$\exp (n W), n$ any integer. Then $N$ is a discrete subgroup of the central of $G$, and so (see [1], p. 12) $G / N$ is a Lie group locally topologically isomorphic with $G$.

But the homomorphism $G \rightarrow G / N$ carries $S_{3}$ into $S_{3} / N$, which is simply isomorphic with $G_{3} / N=G_{3}{ }^{*}$. This and the corollary to Theorem 1 complete the proof.

E. Cartan [5] has shown that the universal covering group of the group of projective transformations of the line is topologically isomorphic in the large with no linear group.

\title{
BIBLIOGRAPHY
}

[1] E. Cartan, Théorie des groupes finis et continus et l'analysis situs, Mémorial des Sciences Mathématiques, no. 42, 1930.

[2] L. P. Eisenhart, Continuous Groups of Transformations, 1933.

[3] W. Mayer and T. Y. Thomas, Foundations of the theory of continuous groups, Annals of Mathematics, vol. 36 (1935), pp. 770-822.

[4] A. Speiser, Gruppentheorie, 2d ed., 1927.

[5] La Topologie des Groupes de Lie, Paris, 1936, p. 18.

Society of Fellows, Harvard University

\section{CHARACTERISTICS OF BIRATIONAL TRANSFORMS IN $S_{r}$}

BY B. C. WONG

1. Introduction. Consider a $k$-dimensional variety, $V_{k}^{n}$, of order $n$ in an $r$-space, $S_{r}$. Let us project $V_{k}^{n}$ from a general $(r-k-t-1)$-space of $S_{r}$ upon a general $(k+t)$-space of $S_{r}$ and denote the projection by ${ }_{t} V_{k}^{n}$. We are supposing that $1 \leqq t \leqq k$. Then upon ${ }_{t} V_{k}^{n}$ lies a double variety, $D_{k-t}$, of dimension $k-t$ and order $b_{t}$ and upon $D_{k-t}$ lies a pinch variety, $W_{k-t-1}$, of dimension $k-t-1$ and order $j_{t+1}$. Since the symbol $W_{-1}$ is without meaning, we thus obtain $2 k-1$ characteristics $b_{1}, b_{2}, \cdots, b_{k}$, $j_{2}, j_{3}, \cdots, j_{k}$. The symbol $j_{1}$ has a meaning which will be explained subsequently.

Now let a general $(r-k+q-2)$-space, $S_{r-k+q-2},(1 \leqq q \leqq k)$, be given in $S_{r}$. Through this $S_{r-k+q-2}$ pass $\infty^{k-q+1}$ primes of $S_{r}$ and $\infty^{k-q}$ of these are tangent to $V_{k}^{n}$. The points of contact form a $(k-q)$-dimensional variety, $U_{k-q}$. Denote its order by $m_{q}$. Thus 
we obtain $k$ further characteristics $m_{1}, m_{2}, \cdots, m_{k}$. If we project $V_{k}^{n}$ upon a $(k+1)$-space, $S_{k+1}$, of $S_{r}$, we see that $m_{q}$ is the class of the $V_{q}^{n}$ in which a $(q+1)$-space of $S_{k+1}$ meets the projected variety. We also say that $m_{q}$ is the class of the $q$-dimensional variety in which an $(r-k+q)$-space of $S_{r}$ meets $V_{k}^{n}$.

In the case where $V_{k}^{n}$ is the complete intersection of $r-k$ general primals, of orders $n_{1}, n_{2}, \cdots, n_{r-k}$, respectively, in $S_{r}$, the values of $j_{t}, b_{t}, m_{q}$ are known* and they are

$$
\begin{aligned}
& j_{t}=n_{1} n_{2} \cdots n_{r-k} \sum\left(n_{1}-1\right)\left(n_{2}-1\right) \cdots\left(n_{t}-1\right), \\
& b_{t}=\frac{1}{2} n_{1} n_{2} \cdots n_{r-k}\left[n_{1} n_{2} \cdots n_{r-k}-1-\sum\left(n_{1}-1\right)\right. \\
& -\sum\left(n_{1}-1\right)\left(n_{2}-1\right)-\cdots \\
& \left.-\sum\left(n_{1}-1\right)\left(n_{2}-1\right) \cdots\left(n_{t}-1\right)\right] \\
& =\frac{1}{2} n_{1} n_{2} \cdots n_{r-k}\left[\sum\left(n_{1}-1\right)\left(n_{2}-1\right) \cdots\left(n_{t+1}-1\right)\right. \\
& +\sum\left(n_{1}-1\right)\left(n_{2}-1\right) \cdots\left(n_{t+2}-1\right)+\cdots \\
& \left.+\sum\left(n_{1}-1\right)\left(n_{2}-1\right) \cdots\left(n_{r-k}-1\right)\right] \text {, } \\
& \text { (III) } m_{q}=n_{1} n_{2} \cdots n_{r-k} \sum_{h} \sum\left(n_{1}-1\right)^{h_{1}}\left(n_{2}-1\right)^{h_{2}} \cdots\left(n_{q}-1\right)^{h_{q}} \text {, }
\end{aligned}
$$

where

$$
h=h_{1}+h_{2}+\cdots+h_{q}=q .
$$

We shall refer to these values later.

In this paper we propose to determine the values of the same characteristics for the variety $V_{k}^{n}$ in $S_{r}$ which we consider as the birational transform of a $k$-dimensional variety, say $\Phi_{k}^{\nu}$, of order $\nu$ in a $\rho$-space, $\Sigma_{\rho}$, for $\rho<r$. We confine ourselves to the case where $\Phi_{k}^{\nu}$ is the complete intersection of $\rho-k$ general primals of $\Sigma_{\rho}$, of respective orders $\nu_{1}, \nu_{2}, \cdots, \nu_{\rho-k}$, given by the equations

$$
F^{(1)}=0, F^{(2)}=0, \cdots, F^{(\rho-k)}=0,
$$

* C. Segre, Mehrdimensionale Räume, Encyklopädie der Mathematischen Wissenschaften, III C7, pp. 944-945. Also B. C. Wong, On certain characteristics of k-dimensional varieties in r-space, this Bulletin, vol. 38 (1932), pp. 725730. 
where $F^{(i)}$ is a homogeneous function of degree $\nu_{i}$ in the variables $\xi_{0}, \xi_{1}, \cdots, \xi_{\rho}$. The order of $\Phi_{k}^{\nu}$ is $\nu=\nu_{1} \nu_{2} \cdots \nu_{\rho-k}$. The corresponding characteristics $\eta_{t}, \beta_{t}, \mu_{q}$ of $\Phi_{k}^{\nu}$ are given by (I), (II), (III) if we replace in the right-hand members $r$ by $\rho$ and $n_{i}$ by $\nu_{i}$.

We suppose that the transformation of $\Phi_{k}^{\nu}$ into $V_{k}^{n}$ is accomplished by means of a general linear $\infty r_{\text {-system, }}|\psi|$, without base varieties of any kind, of $(k-1)$-dimensional varieties of order $\nu N$, and that $|\psi|$ is the intersection of $\Phi_{k}^{\nu}$ and a general linear $\infty r_{\text {-system, }}|\phi|$, of primals of order $N$, none passing through $\Phi_{k}^{\nu}$, given by the equation

$$
a_{0} \phi^{(0)}+a_{1} \phi^{(1)}+\cdots+a_{r} \phi^{(r)}=0,
$$

the $\phi$ 's being linearly independent homogeneous polynomials of degree $N$ in the $(\rho+1) \quad \xi$ 's. Then, the order of $V_{k}^{n}$ is $n=\nu N^{k}=\nu_{1} \nu_{2} \cdots \nu_{\rho-k} N^{k}$. The coordinates of the points on $V_{k}^{n}$ are given by

$$
\begin{gathered}
\sigma x_{0}=\phi^{(0)}\left(\xi_{0}, \xi_{1}, \cdots, \xi_{\rho}\right), \quad \sigma x_{1}=\phi^{(1)}\left(\xi_{0}, \xi_{1}, \cdots, \xi_{\rho}\right), \\
\cdots, \quad \sigma x_{r}=\phi^{(r)}\left(\xi_{0}, \xi_{1}, \cdots, \xi_{\rho}\right),
\end{gathered}
$$

where the $\xi$ 's satisfy equations (1).

It is to be noted that an $h$-dimensional locus of order $l$ on $\Phi_{k}^{\nu}$ goes into an $h$-dimensional locus of order $l N^{h}$ on $V_{k}^{n}$. For $h=k, \Phi_{k}^{\nu}$ goes into $V_{k}^{n}$, where $n=\nu N^{k}$.

We shall first, in $\S 2$, derive a general relation connecting the $b$ 's and the $j$ 's for a general variety which has no extraordinary singular points. The determination of the values of the $m$ 's of our variety $V_{k}^{n}$ will be given in $\S 3$ and the determination of those of the $j$ 's in $\S 4$. The values of the $b$ 's will then be obtained with the aid of the relation derived in $\$ 2$. Incidentally, we find it interesting to express the $m$ 's and $j$ 's in terms of the $\mu$ 's and $\eta$ 's, respectively, of $\Phi_{k}^{\nu}$.

2. The Relation between the b's and the j's. Let $C^{l}$ be a curve of order $l$, in a space of dimension greater than 2, whose points are paired in an (irrational) involution $I_{2}$. Suppose that $C^{l}$ has $d$ actual nodes at each of which two corresponding points of $I_{2}$ coincide but lie on different branches of the curve. If $i$ denotes the number of simple points of $\mathrm{C}^{l}$ at each of which two corresponding points of $I_{2}$ become united, the order of the ruled sur- 
face, which may be a cone, whose generators are lines joining corresponding points of the involution is, as is well known,

$$
R=(2 l-i-2 d) / 2 \text {. }
$$

Now consider a general $k$-dimensional variety $V_{k}$, of any order $n$, without extraordinary singular points, in $S_{r}$ and let it be intersected by a general $(r-k+t)$-space of $S_{r}$ in a $V_{t}$. If we project $V_{t}$ upon a $(2 t-1)$-space, $S_{2 t-1}$, we see that the projection ${ }_{t-1} V_{t}$ has a double curve $D_{1}$ of order $b_{t-1}$ and $j_{t}$ pinch points. This $t_{t-1} V_{t}$ may certainly be regarded as the projection of a ${ }_{t} V_{t}$ in a $(2 t)$-space, $S_{2 t}$, the ${ }_{t} V_{t}$ being assumed to be a projection of $V_{t}$. Let $Z$ be the point, taken in a general position of $S_{2 t}$, from which ${ }_{t} V_{t}$ is projected into ${ }_{t-1} V_{t}$ in $S_{2 t-1}$. There are $\infty^{1}$ lines through $Z$ meeting ${ }_{t} V_{t}$ in two distinct points and the locus of these lines is a ruled surface, in fact a cone, of order $b_{t-1}$. This cone meets ${ }_{t} V_{t}$ in a curve $c$ of order $2 b_{t-1}$, of which the double curve $D_{1}$ on ${ }_{t-1} V_{t}$ is the projection. The curve $c$ has $b_{t}$ actual nodes which are the improper double points of ${ }_{t} V_{t}$. There are $j_{t}$ elements of the cone tangent to ${ }_{t} V_{t}$ and also to $c$. The projections of the points of contact are the pinch points on ${ }_{t-1} V_{t}$. Now on $c$ is an involution of pairs of points set up by the elements of the cone. There are $b_{t}$ points each of which is the union of two corresponding points on different branches of the curve and $j_{t}$ points each of which is the union of two corresponding points on a simple branch of the curve. Putting $R=b_{t-1}, l=2 b_{t-1}, i=j_{t}, d=b_{t}$ in the relation of the paragraph just preceding, we have the desired relation $b_{t-1}=\left(4 b_{t-1}-j_{t}-2 b_{t}\right) / 2$, or

$$
2 b_{t-2}=j_{t}+2 b_{t} \text {. }
$$

By letting $t=1,2, \cdots, k$, successively, we obtain

$$
2 b_{0}=j_{1}+2 b_{1}, 2 b_{1}=j_{2}+2 b_{2}, \cdots, \quad 2 b_{k-1}=j_{k}+2 b_{k} .
$$

As we shall see presently, $b_{0}=n(n-1) / 2$ and $j_{1}$ is identical with the class $m_{1}$ of a plane section of the projection ${ }_{1} V_{k}$ in a $(k+1)$ space. The relation (3) may be replaced by the relation

$$
2 b_{s}-2 b_{t}=j_{s+1}+j_{s+2}+\cdots+j_{t}, \quad(s>t) .
$$

Note that this relation, or relation (3), is satisfied by (I) and (II). 
3. The Determination of the $m$ 's. Returning to the $V_{k}^{n}$ which is the birational transform of $\Phi_{k}^{\nu}$ in $\Sigma_{\rho}$, we see at once that $m_{q},(1 \leqq q \leqq k)$, is the order of the variety $U_{k-q}$ on $V_{k}^{n}$ which has for image on $\Phi_{k}^{\nu}$ the complete intersection $\Theta_{k-q}$ of $\Phi_{k}^{\nu}$ and the Jacobian variety $\omega_{\rho-q}$ of the $\rho-k$ primals, given by (1), intersecting in $\Phi_{k}^{\nu}$, and any $k-q+2$ independent primals of the system $|\phi|, \operatorname{say} \phi^{(1)}=0, \phi^{(2)}=0, \cdots, \phi^{(k-q+2)}=0 . \Theta_{k-q}$ is the locus of the points of contact between $\Phi_{k}^{\nu}$ and the $\infty^{k-q}$ primals of the $\infty^{k-q+1}$-system determined by the $k-q+2$ primals just mentioned which are tangent to $\Phi_{k}^{\nu}$. The conditions of contact are given by

$$
\left\|\begin{array}{cccc}
F_{0}^{(1)} & F_{1}^{(1)} & \cdots F_{\rho}^{(1)} \\
\cdot & \cdot & \cdots & \cdot \\
F_{0}^{(\rho-k)} & F_{1}^{(\rho-k)} & \cdots F_{\rho}^{(\rho-k)} \\
\phi_{0}^{(1)} & \phi_{1}^{(1)} & \cdots \phi_{\rho}^{(1)} \\
\cdot & \cdot & \cdots & \cdot \\
\phi_{0}^{(k-q+2)} & \phi_{1}^{(k-q+2)} & \cdots \phi_{\rho}^{(k-q+2)}
\end{array}\right\|=0
$$

where $F_{i}{ }^{(h)}, \phi_{i}{ }^{(h)}$ are written in place of $\partial F^{(h)} / \partial \xi_{i}, \partial \phi^{(h)} / \partial \xi_{i}$, respectively. This equality represents the Jacobian $\omega_{\rho-q}$. The matrix being of $\rho+1$ columns and $\rho-q+2$ rows, the order of $\omega_{\rho-q}$ is $*$

$$
\begin{aligned}
H_{q}= & \sum_{i=0}^{q}\left(\begin{array}{c}
k+1-i \\
q-i
\end{array}\right)(N-1)^{q-i} \\
& \cdot \sum_{i} \sum\left(\nu_{1}-1\right)^{h_{1}\left(\nu_{2}-1\right)^{h_{2}} \cdots\left(\nu_{i}-1\right)^{h_{i}}}
\end{aligned}
$$

where

$$
h_{1}+h_{2}+\cdots+h_{i}=i \text {. }
$$

Then the order of $\Theta_{k-q}$ is $\nu_{1} \nu_{2} \cdots \nu_{\rho-k} H_{q}$ and therefore the order of $U_{k-q}$ on $V_{k}^{n}$ which is the transform of $\Theta_{k-q}$ is

$$
m_{q}=\nu_{1} \nu_{2} \cdots \nu_{\rho-k} N^{k-q} H_{q} .
$$

It is of interest to express $m_{q}$ in terms of the $\mu$ 's belonging to $\Phi_{k}{ }^{\nu}$. The various values of the $\mu$ 's are obtained from (III) by changing $r$ to $\rho$ and $n_{i}$ to $\nu_{i}$. By taking account of (5), we have, from (6), writing $\mu_{0}$ in place of $\nu$ or $\nu_{1} \nu_{2} \cdots \nu_{\rho-k}$,

* Salmon, Modern Higher Algebra, 4th ed., Lesson 19. 


$$
\begin{aligned}
& m_{q}= N^{k-q} \sum_{i=0}^{q}\left(\begin{array}{c}
k+1-i \\
q-i
\end{array}\right)(N-1)^{q-i} \mu_{i} \\
&=N^{k-q}\left[\left(\begin{array}{c}
k+1 \\
q
\end{array}\right)(N-1)^{q} \mu_{0}\right. \\
&\left.\quad+\left(\begin{array}{c}
k \\
q-1
\end{array}\right)(N-1)^{q-1} \mu_{1}+\cdots+\mu_{q}\right] .
\end{aligned}
$$

4. The Determination of the $j$ 's and the b's. The projection ${ }_{t-1} V_{k}^{n}$, in a $(k+t-1)$-space of $S_{r}$, of $V_{k}^{n}$ has a pinch variety $W_{k-t}$ of order $j_{t}$. This $W_{k-t}$ has for image on $\Phi_{k}^{\nu}$ the complete intersection of $\Phi_{k}^{\nu}$ and the variety $\pi_{\rho-t}$ common to all the primals each of which is the Jacobian of $\rho+1$ of the following $\rho+t$ primals:

$$
\begin{array}{llll}
F^{(1)}=0, & F^{(2)}=0, & \cdots, & F^{(p-k)}=0, \\
\phi^{(1)}=0, & \phi^{(2)}=0, & \cdots, & \phi^{(k+t)}=0 .
\end{array}
$$

The $k+t$ primals represented by the $\phi$ 's equated to zero are supposed to be independent members of the system $|\phi|$ given by (2). The equations of $\pi_{\rho-t}$ are

$$
\left\|\begin{array}{cccc}
F_{0}^{(1)} & F_{1}^{(1)} & \cdots & F_{\rho}^{(1)} \\
\cdot & \cdot & \cdot & \cdot \\
F_{0}^{(\rho-k)} & F_{1}^{(\rho-k)} & \cdots & F_{\rho}^{(\rho-k)} \\
\phi_{0}^{(1)} & \phi_{1}^{(1)} & \cdots & \phi_{\rho}^{(1)} \\
\cdot & \cdot & \cdot & \cdot \\
\phi_{0}^{(k+t)} & \phi_{1}^{(k+t)} & \cdots & \phi_{\rho}^{(k+t)}
\end{array}\right\|=0 .
$$

The left-hand member being a matrix of $\rho+t$ rows and $\rho+1$ columns, the order of $\pi_{\rho-t}$ is*

$$
\begin{aligned}
C_{t} & =\sum_{i=0}^{t}\left(\begin{array}{c}
k+t \\
i
\end{array}\right)(N-1)^{i} \sum\left(\nu_{1}-1\right)\left(\nu_{2}-1\right) \cdots\left(\nu_{t-i}-1\right) \\
& =\sum_{i=0}^{t}\left(\begin{array}{c}
k+t \\
t-i
\end{array}\right)(N-1)^{t-i} \sum\left(\nu_{1}-1\right)\left(\nu_{2}-1\right) \cdots\left(\nu_{i}-1\right),
\end{aligned}
$$

* Salmon, loc. cit. 
and the order of the intersection of $\Phi_{k}^{\nu}$ and $\pi_{\rho-t}$ is $\nu C_{t}$. Therefore, the order of $W_{k-t}$ is

$$
\begin{aligned}
& j_{t}=\nu N^{k-t} C_{t} \\
&=\nu_{1} \nu_{2} \cdots \nu_{\rho-k} N^{k-t} \sum_{i=0}^{t}\left(\begin{array}{c}
k+t \\
t-i
\end{array}\right)(N-1)^{t-i} \\
& \cdot \sum\left(\nu_{1}-1\right)\left(\nu_{2}-1\right) \cdots\left(\nu_{i}-1\right) .
\end{aligned}
$$

Thus, for $t=1$, we have

$$
j_{1}=\nu_{1} \nu_{2} \cdots \nu_{\rho-k} N^{k-1}\left[(k+1)(N-1)+\sum\left(\nu_{1}-1\right)\right]=m_{1} .
$$

Now in terms of the $\eta$ 's of $\Phi_{k}^{\nu}$ we have, from (I) and (8),

$$
\begin{aligned}
j_{t}=N^{k-t} \sum_{i=0}^{t}\left(\begin{array}{c}
k+t \\
t-i
\end{array}\right)(N-1)^{t-i} \eta_{i} & \\
=N^{k-t}\left[\left(\begin{array}{c}
k+t \\
t
\end{array}\right)(N-1)^{t} \eta_{0}\right. & +\left(\begin{array}{c}
k+t \\
t-1
\end{array}\right)(N-1)^{t-1} \eta_{1}+\cdots \\
& \left.+\left(\begin{array}{c}
k+t \\
1
\end{array}\right)(N-1) \eta_{t-1}+\eta_{t}\right]
\end{aligned}
$$

where $\eta_{0}=\nu=\nu_{1} \nu_{2} \cdots \nu_{\rho-k}$.

To determine the $b$ 's we make use of the relation (3) or (4) of $\$ 2$. A little calculation yields

$$
b_{t}=\frac{1}{2} \nu\left[\nu N^{2 k}-\sum_{h=0}^{t} N^{k-h} C_{h}\right],
$$

where $C_{h}$ is given by (7) if we replace in it $t$ by $h$. Since any $(k-t)$-dimensional variety of order $l$ on $\Phi_{k}^{\nu}$ goes into a $(k-t)$ dimensional variety of order $l N^{k-t}$ on $V_{k}^{n}$, we see that

$$
\frac{2 b_{t}}{N^{k-t}}=\nu^{2} N^{k+t}-\nu \sum_{h=0}^{t} N^{t-h} C_{h}
$$

is the order of the image on $\Phi_{k}^{\nu}$ of the double variety $D_{k-t}$ on the projection ${ }_{t} V_{k}^{n}$ in a $(k+t)$-space.

The University of California 